\title{
The percutaneous coronary intervention prior to transcatheter aortic valve implantation (ACTIVATION) trial: study protocol for a randomized controlled trial
}

\author{
Muhammed Zeeshan Khawaja ${ }^{1,2^{* \dagger}}$, Duolao Wang $^{3 \dagger}$, Stuart Pocock ${ }^{4 \dagger}$, Simon Robert Redwood ${ }^{1,2 \dagger}$ \\ and Martyn Rhys Thomas ${ }^{2+}$
}

\begin{abstract}
Background: Current guidelines recommend treatment of significant coronary artery disease by concomitant coronary artery bypass grafting $(C A B G)$ in patients undergoing surgical aortic valve replacement. However there is no consensus as to how best to treat coronary disease in high-risk patients requiring transcatheter aortic valve implantation (TAVI).

Methods/Design: The percutaneous coronary intervention prior to transcatheter aortic valve implantation (ACTIVATION) trial is a randomized, controlled open-label trial of 310 patients randomized to treatment of significant coronary artery disease by percutaneous coronary intervention (PCl - test arm) or no PCl (control arm). Significant coronary disease is defined as $\geq 1$ lesion of $\geq 70 \%$ severity in a major epicardial vessel or $50 \%$ in a vein graft or protected left main stem lesion. The trial tests the hypothesis that the strategy of performing pre-TAVI PCI is non-inferior to not treating such coronary stenoses with $\mathrm{PCI}$ prior to TAVI, with a composite primary outcome of 12-month mortality and rehospitalization. Secondary outcomes include efficacy end-points such as 30-day mortality, safety endpoints including bleeding, burden of symptoms, and quality of life (assessed using the Seattle Angina Questionnaire and the Kansas City Cardiomyopathy Questionnaire).

In conclusion, we hope that using a definition of coronary artery disease severity closer to that used in everyday practice by interventional cardiologists - rather than the $50 \%$ severity used in surgical guidelines - will provide robust evidence to direct guidelines regarding TAVI therapy and improve its safety and efficacy profile of this developing technique.
\end{abstract}

Trial registration: ISRCTN75836930, http://www.controlled-trials.com/ISRCTN75836930 (registered 19 November 2011).

Keywords: Transcatheter aortic valve implantation, Percutaneous coronary intervention, Aortic stenosis, Coronary

\section{Background}

Transcatheter aortic valve implantation (TAVI) has moved into the cardiology mainstream with rapid acceptance of this new technology since the first implant in 2002 [1]. The two devices with the largest experiences are the

\footnotetext{
* Correspondence: Dr.zeeshan.khawaja@gmail.com

${ }^{\dagger}$ Equal contributors

'The Rayne Institute, Cardiovascular Division, King's College London, BHF Centre of Excellence, St Thomas' Hospital, Westminster Bridge Road, London SE1 7EH, UK

2Department of Cardiology, Guy's \& St Thomas' NHS Foundation Trust, Westminster Bridge Road, London SE1 7EH, UK

Full list of author information is available at the end of the article
}

self-expanding CoreValve Revalving ${ }^{\mathrm{TM}}$ system (Medtronic CoreValve, Luxembourg) and the balloon expandable Edwards Sapien XT valve (Edwards Lifesciences, Irvine, CA). Both are employed in patients whose peri-operative risk is deemed too high for surgical aortic valve replacement (sAVR). Coronary artery disease has a high prevalence in these patients [2-5] and shares many of the same causative factors [6]. While the matrices for these devices state that significant coronary artery disease $(C A D)$ should preferably be treated prior to implantation, current practice is frequently not to do so. Indeed, successful PCI has been demonstrated post-implantation [7-9], as both technologies 
allow access to the coronary ostia. The presence of concomitant $\mathrm{CAD}$ has been associated with adverse procedural outcomes in sAVR [10,11] and now also in TAVI [5]. In this higher risk cohort, with a mean age in excess of the 'normal' population with chronic stable angina, we must carefully evaluate any coronary artery lesion's significance in terms of the possibly greater risks of PCI and the impact on the planned valvular intervention.

\section{Considerations in pre-transcatheter aortic valve implantation percutaneous coronary intervention}

Among the possible advantages of revascularization prior to TAVI may be a protective effect against the ischemic burden of the procedure, including as it does periods of hypotension. The absence of contractile reserve is associated with increased mortality after sAVR [12], and significant stenoses not intervened upon could contribute to this. Surgical revascularization for multi-vessel coronary artery disease has been found to be an independent factor predictive of improvement of left ventricular ejection fraction (LVEF) after sAVR [13], and similar benefits for revascularization by PCI may exist. Improving coronary flow in symptomatic patients with significant flow-limiting stenoses may maximize this beyond the valvular intervention. Wave intensity analysis of coronary flow has demonstrated marked reduction in the diastolic suction wave in aortic stenosis (the dominant wave in coronary perfusion [14]), which significant stenosis may impair further [15].

The risks of PCI are well described: death, myocardial infarction, coronary artery bypass grafting (CABG), stroke, vascular access complications, renal insufficiency, allergy and stroke/TIA $[16,17]$. PCI is also associated with an incidence of stent thrombosis of up to $1 \%$ with significant mortality $[18,19]$. The presence of severe aortic stenosis could have a detrimental effect upon the ability to withstand these. Indeed, the hypotension experienced by patients during the TAVI procedure (especially during rapid right ventricular pacing) may actually increase the risk of stent thrombosis. There has been increasing recognition recently of the adverse outcomes associated with major bleeding post-PCI [20,21]. Current opinion is to continue aspirin for life and clopidogrel for one month after bare metal stenting and 12 months after insertion of a drug-eluting stent. This would possibly impact upon bleeding complications in transfemoral, subclavian and transaortic TAVI and would certainly be of concern in patients undergoing TAVI via the transapical approach, given the direct myocardial access required. Angiography in the 24 hours, or even the 5 days, prior to sAVR has been shown to be associated with acute kidney injury post-operatively [22]. These risks may also exist if PCI and TAVI are inadequately separated or combined in a hybrid procedure. Finally, patients would require two admissions with attendant implications to cost and risk. This latter consideration might be offset against the possible reduction in length of stay in the post-TAVI period.

\section{Why do we need a randomized trial?}

The data on coronary artery disease in TAVI and PCI in this context is certainly provocative, but there are several major limitations, given that the studies are all nonrandomized, registry-type with relatively small sample sizes.

The lack of data on pre-procedural revascularization has led to some variability between centers on the level of coronary interventions undertaken prior to implantation. A retrospective analysis by Masson et al. stratified TAVI patients according to myocardium at risk due to coronary artery disease, finding no difference in 30-day or 1-year mortality between the groups [4]. However the small group sizes involved would have required a mortality difference between even the two most extreme groups at 30 days of greater than one third to gain significance, limiting our use of this data. The access approach used was not included in their risk model, an important omission given the known differences in risk profiles between, for example, TF-TAVI and TA-TAVI cohorts. In the German TAVI registry, CAD was defined as previous revascularization (either PCI or CABG) OR a stenosis of $\geq 50 \%$ and was noted in $62 \%$ of 1,382 patients. These patients had a greater in-hospital mortality (10.0 versus $5.5 \%, P<0.01)$, required more frequent cardio-pulmonary resuscitation $(7.8$ versus $3.5 \%, P<0.01$ ) and suffered greater 30 -day mortality ( $\log \operatorname{rank} P=0.041)$. But there were significant differences in the group demographics: more frequent peripheral vascular disease, lower left ventricular (LV) ejection fraction and higher logistic EuroScores in patients with CAD [23]. The Italian CoreValve registry noted that in patients with critical ostial disease, myocardial infarction (MI) within 12 months of TAVI was greater in those patients who were not revascularized prior to TAVI [24]. A recent meta-analysis of the effects of CAD upon outcome in the midterm showed no effect; but again, of seven included studies, five defined $\mathrm{CAD}$ as previous revascularization, and the remaining two used 50\% stenosis severity [25].

The presence of pre-existing treated coronary artery disease has been identified as an adverse risk factor for procedural and long term outcome [5]. With regards to the effects of pre-TAVI PCI, Masson and colleagues performed a sub-analysis of just 15 patients in whom PCI was performed at the discretion of the individual cardiologist: the resultant selection bias in turn clouds the interpretation of any findings. The mortality in the overall group one-year after TAVI was reported as $77.9 \%$ and was found to be $80 \%$ in those with pre-emptive PCI - though no comparison between the two cohorts was performed [4]. Abdel Wahab et al. reported that 55 patients who underwent PCI prior to TAVI showed no 
difference in adverse events at either 30 days (2\% versus $6 \% ; P=0.27$ ) or 6 months (9\% versus $14 \% ; P=0.42$ ) when compared to a group undergoing isolated TAVI [26]. The feasibility of PCI for CAD in patients with severe AS has been demonstrated by Goel et al. in a cohort of 258 patients over a 10-year period with a favorable comparison with propensity matched patients without the aortic disease [27].

There has been a rapid, global expansion in the use of TAVI to treat aortic stenosis in patients who are not candidates for sAVR. The efficacy of this technique has been successfully demonstrated in randomized, controlled trials [28,29] and in large registries [30,31], and there is now a need to improve patient safety and outcome, including how to manage patients with concomitant CAD. Given the paucity of data on the issue and variation in practice and recommendations we feel a randomized controlled trial (RCT) is essential for safe evidence-based practice. ACTIVATION is the first randomized controlled trial of elective PCI prior to TAVI. Its findings will help define the optimum revascularization strategy in this procedure and help create evidence-based guidelines on this controversial issue.

\section{Methods/Design}

\section{Hypothesis, design and end points}

ACTIVATION is a prospective, randomized, open-label trial that aims to test the hypothesis that revascularization of significant coronary artery disease by PCI prior to TAVI (Test arm) will result in a rate of mortality and rehospitalization at 12 months that is non-inferior to TAVI without such revascularization (control). Because the control patients in this study are receiving a CE/FDA approved valve replacement, a trial arm comparison would not be likely to demonstrate superiority in either direction.

The trial has an intentional non-inferiority design. The safety of TAVI with revascularization performed as deemed necessary by the Heart Team has been demonstrated in previous trials and registries [29,30,32]. From the available data detailed in the statistical design section, our hypothesis is that the no PCI strategy will have comparable effectiveness to the PCI (the active control) strategy, hopefully helping the cardiology community decide whether the extra procedure of pre-TAVI PCI can be safely avoided. From an ethical standpoint, a sham procedure in the no PCI arm could not be countenanced. Given the complexities of the TAVI procedure and the recommended Heart Team approach, we could not blind operators as to whether patients had undergone revascularization or not. To minimize bias towards the null, we have robust inclusion/exclusion criteria and clear end-point definitions and boundaries. Any deviation from the protocol in delivery of strategy will be carefully recorded, including any concomitant therapies.

\section{Patient population}

Patient eligibility will be confirmed by the study investigators according to the inclusion and exclusion criteria in Table 1.

\section{Primary endpoint}

The primary endpoint is a composite of mortality and rehospitalization at 12 months since randomization. Mortality is defined as death due to any cause, the exact nature and date of which will be recorded. All deaths will be considered cardiovascular-related unless there is documentation to the contrary.

\section{Secondary endpoints}

These consist of:

i) Mortality at 30 days and 12 months post-TAVI

ii) Major adverse cardiovascular or cerebrovascular event (MACCE) at 30 days and 12 months

iii) Acute myocardial infarction (MI) at 30 days and 12 months

iv) Stroke at 30 days and 12 months

v) Repeat revascularization by either PCI or CABG

vi) Hospitalization for heart failure at 30 days and 12 months

vii) Procedural complications (ventricular fibrillation (VF) or ventricular tachycardia (VT) requiring cardioversion

viii) Requirement of cardiopulmonary bypass

ix) Cardiopulmonary arrest requiring cardiopulmonary resuscitation (CPR) and/or assisted ventilatory support

x) Procedural success

xi) Bleeding complications

xii) Access site complications

xiii) Cerebrovascular events

xiv) Acute kidney injury

$\mathrm{xv)}$ Duration of hospital stay

xvi) Quality of life

xvii) Anginal burden

xviii) 6-minute walk test

The definitions are in accordance with the Valve Academic Research Consortium (VARC) guidelines and its update and are specified in Table 2 [33].

\section{Ethics}

Ethics review was approved by United Kingdom National Research Ethics Committee (London Dulwich) (11/LO/ 1596).

\section{Randomization}

Randomization will take place once the patient has provided written, informed consent. This will be performed 
Table 1 Eligibility criteria for the percutaneous coronary intervention prior to transcatheter aortic valve implantation (ACTIVATION) trial

Inclusion criteria

Definition

- Patients $\geq 18$ years of age

- Severe aortic stenosis, as defined by

- Symptoms suggestive of aortic stenosis

- Deemed of prohibitive risk for open aortic valve replacement by a TAVI multidisciplinary team (MDT) and accepted for TAVI by said TAVI MDT.

Significant coronary artery disease

- Undergoing TAVI via any accepted approach

- Written informed consent

\section{Exclusion Criteria}

- Recent acute coronary syndrome

- Unprotected left main stem disease

- Significant angina

- Pregnancy

- Active internal bleeding (except menstruation)

- Allergy to heparin or GPIlb/llla inhibitors

- Thrombocytopenia cells $/ \mathrm{mm}^{3}$ )

- Patients who have previously been enrolled in this study

- Patients who are currently enrolled in any other study where involvement in

ACTIVATION would involve deviation from either protocol

TAVI, transcatheter aortic valve implantation; CCS, Canadian Cardiac Society; Gpllb/llla, glycoprotein Ilb/llla

via an online portal. Randomization of the treatment assignment is based on the block method using randomly varying block sizes and will be stratified by center. Once the patient is randomized $s /$ he will be monitored for the duration of the follow-up irrespective of subsequent clinical management. The intended timeframe for PCI should be stated at randomization. After PCI the patient should receive dual anti-platelet therapy for 30 days - one of which can then stop if the patient receives a bare-metal stent (BMS). All PCI patients should undergo TAVI within the 2 weeks after this 30-day post-PCI time point. Any clinical events which occur in the test arm during this period will be attributed to the percutaneous revascularization on an intention-to-treat-basis. The flow diagram in Figure 1 illustrates the recruitment and study process.

\section{Trial conduct}

ACTIVATION will be conducted at up to 20 hospitals in Europe providing TAVI using the Edwards system. Trial administration and data management will be carried out by an independent clinical trial organization,
- Peak transvalvular gradient of $\geq 40 \mathrm{mmHg}$ on transthoracic echocardiography (TTE), transesophageal echocardiography (TOE) or dobutamine stress echocardiography (DSE)

- Aortic valve area of $<1.0 \mathrm{~cm}^{2}$

Dyspnea, syncope etc. but not pre-dominantly angina

- $\geq 1$ stenosis of $\geq 70 \%$ in a major epicardial artery deemed suitable for $\mathrm{PCl}$ ( $\geq 50 \%$ if protected left main stem or vein graft)

Transfemoral, transapical, subclavian or transaortic

Definition

Within 30 days of randomization

CCS class $\geq 3$

Platelet count $<100,000$ the European Cardiovascular Research Center, Massy, France. The trial is registered at www.controlled-trials. com (ISRCTN75836930).

\section{Procedural details}

\section{Percutaneous coronary intervention}

The percutaneous coronary intervention in the test arm should be performed according to current local best practice. The use of the OMEGA bare metal stent (Boston Scientific Inc, Natick, MA, USA) or PROMUS family of drug-eluting stents (DES; Boston Scientific Inc, Natick, MA, USA) is mandatory. It is strongly recommended that patients should be pre-treated with aspirin $300 \mathrm{mg}$ and either clopidgrel $600 \mathrm{mg}$, prasugrel $60 \mathrm{mg}$, or ticagrelor $180 \mathrm{mg}$ as per local guidelines. Patients should also receive appropriate anticoagulant therapy during the PCI. The route of access (radial or femoral), use of further interventional techniques (for example, intravascular ultrasound, rotablation, laser atherectomy etcetera) and PCI equipment are at the discretion of the operator. Post-procedure aspirin should be continued at a maintenance of $75 \mathrm{mg}$ o.d.. Patients should receive either 
Table 2 Endpoint definitions

\begin{tabular}{ll}
\hline End-point & Definition \\
\hline Mortality & All-cause death \\
Cardiovascular mortality & $\begin{array}{l}\text { All deaths unless otherwise clearly } \\
\text { documented to be related to } \\
\text { another cause (for example, trauma, } \\
\text { cancer, suicide etcetera) }\end{array}$
\end{tabular}

Myocardial infarction (MI) Peri-procedural MI related to TAVI ( $\leq 72 \mathrm{~h}$ after the TAVI)

Spontaneous Ml (>72 h after TAVl)

$\mathrm{Ml}$ related to $\mathrm{PCl}$ (type 4a)

$\mathrm{Ml}$ related to $\mathrm{PCl}$ due to stent thrombosis (type 4b)

Cerebrovascular event Stroke

Transient ischemic attack

Bleeding complication

Life-threatening or disabling

Major

Minor

Access site complication Major

Minor

Acute kidney injury As per Stage 1

AKIN classification in VARC2 [34] Stage 2

Stage 3

Major adverse cardiac and cerebrovascular events (MACCE)

TAVI, transcatheter aortic valve implantation; $\mathrm{PCl}$, percutaneous coronary intervention, AKIN, acute kidney injury network; VARC2, Valve Academic Research Consortium second guidelines [34].

clopidogrel $75 \mathrm{mg}$ o.d., Prasugrel $10 \mathrm{mg}$ o.d. or Ticagrelor $90 \mathrm{mg}$ b.d. for 30 days after PCI for BMS or 6 months for DES as per local guidelines.

\section{Transcatheter aortic valve implantation}

Patients should be accepted for TAVI by a multidisciplinary team including both an interventional cardiologist and a cardiothoracic surgeon. The procedure should use the balloon-expandable Edwards SAPIEN XT prosthesis (Edwards Lifesciences, Irvine, CA, USA). The choices of prosthesis size and access route (transfemoral, transapical or transaortic) are to be left to the discretion of the operating team. Equally the intraprocedural details (local versus general anesthetic, perioperative imaging etcetera) should proceed as per usual practice at the enrolling center. Both aspirin and clopidogrel should be continued for at least 6 months after TAVI implantation with lifelong single anti-platelet therapy thereafter.

\section{Data collection and monitoring}

Data will be collected at baseline from enrolled patients including demographics, past medical history, previous cardiac investigations and current medication. Patients will undergo transthoracic \pm transesophageal echocardiography at baseline, post-procedure, 1 month and 12 months. Twelve-lead electrocardiograms will be obtained at the same time points, with additional traces in the test arm pre- and post-PCI and in either arm after any clinical event thought to represent myocardial infarction and before/after any subsequent revascularization procedure. In addition to routine blood tests, a full blood count will be taken pre- and post-PCI.

Prospective monitoring of adverse and clinical events starts at randomization and continues until 12 months. Patients should be followed up to hospital discharge following PCI. All MACCE will be documented by the research coordinator using the clinical event data form. The same applies for the TAVI procedure. Patients will be followed up at 30 days and 12 months for anginal burden using the Seattle Angina Questionnaire and other end-points. Mortality data will be obtained from the Office of National Statistics in England and Wales, the General Register Office in Scotland and from the appropriate authority in other countries.

All MACCE and other serious adverse events will be recorded in the electronic Case Record Form and reported to the coordinating center within 3 working days of first identification. On receipt of notification of any trial adverse or clinical event, the coordinating center will request additional details, specific to the nature of the event. These episodes will be carefully monitored by the trial coordinator and will form part of the information provided at regular intervals to the Clinical Events and Data Monitoring Committees. The study site will also notify their ethics committee and institutional clinical risk management team according to their local policy. The Clinical Events Committee will be blinded to treatment and will consider each MACCE or adverse event reported and ratify occurrence of an endpoint according to the study definitions (according to a majority).

\section{Statistical consideration \\ Sample size determination}

No randomized trials have been performed of elective PCI versus no PCI in patients undergoing TAVI. In a recent nonrandomized registry, Masson et al. reported a 1 -year mortality of $20 \%$ in 15 patients who underwent PCI prior to TAVI - similar to those who did not at $22.1 \%$ [4] - despite the presence of existing coronary disease having been shown to increase mortality in patients undergoing TAVI from 18.4 to $35.7 \%$ [5].

With the postulation that improved coronary perfusion may protect against some of the ischemic/hypotensive procedural complications of the TAVI procedure, an improvement in rate of mortality seems likely. PCI may 


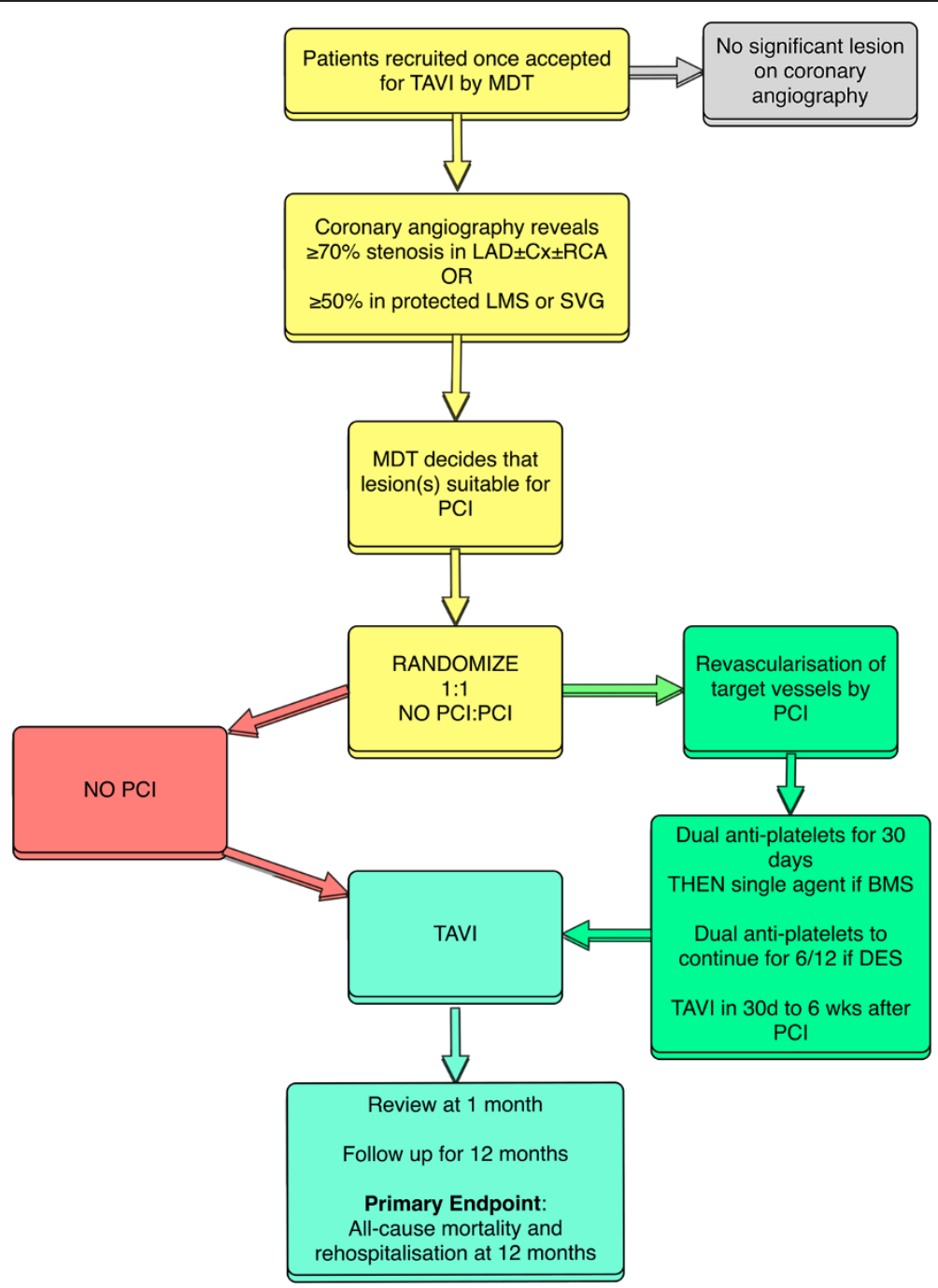

Figure 1 Flow chart. (TAVI, transcatheter aortic valve implantation; MDT, multidisciplinary team; LAD, left anterior descending artery; $C x$, circumflex artery; RCA, right coronary artery; LMS, left main stem; SVG, saphenous vein graft; PCl, percutenaous coronary intervention; BMS, bare metal stent; DES, drug eluting stent).

lead to restenosis of the stent, requiring rehospitalization in $5 \%$ of patients over 12 months. The rate of rehospitalization with stable angina, acute coronary syndrome or ischemia-related heart failure requiring treatment, including possible PCI, is thought to be in the region of $15 \%$ in all patients with CAD. The feasibility assumptions for 12-month mortality are 30\% in the control (TAVI only) arm and $20 \%$ in the test (TAVI + PCI) arm, with similar rates of hospitalization at $15 \%$ in both. Based on the above assumptions, we performed sample size simulations and the simulated results show that with a non-inferiority margin of $7.5 \%$ and a loss to follow up of 5\%, the sample size of 310 patients will have at least $88.8 \%$ power to demonstrate non-inferiority of TAVR following PCI compared with TAVR without PIC in the primary endpoint.

\section{Statistical analysis}

For the primary endpoint analysis, cumulative event rate will be calculated using the Kaplan-Meier estimates, and the Greenwood standard errors for these estimates. A 95\% one-sided upper confidence limit will be computed for the difference in the primary composite outcome of mortality of rehospitalization at 12 months between arms (Test - Control). The Test arm (PCI) will be judged not inferior to the Control arm (no PCI) if the upper confidence limit is less than $7.5 \%$ - the predetermined non-inferiority margin. This was chosen based upon expert opinion and precedent (PARTNER cohort A [29]). In addition to formal analysis of non-inferiority endpoints, the Kaplan-Meier curves will be presented and compared by log rank test by treatment group and hazard ratio and its $95 \%$ confidence interval will be calculated using Cox 
regression model. Also, the combination of the all cause mortality and time to first recurrent hospitalization will be analyzed using the unmatched win ratio approach [35].

For secondary time-to-event outcomes, event rates at 30 and 365 days will be calculated using the Kaplan-Meier method and compared by log rank test. Their hazard ratios will be estimated using Cox regression model. Outcomes with repeated measurements will be analyzed using generalized linear mixed models. Continuous variables will be summarized using number of observations, median (interquartile range) or mean (SD) depending on variable distributions, whereas categorical variables will be summarized by the number and percentage of events. Chi-square tests and Mann-Whitney methods will also be used for comparative purposes. All analyses will be performed both on intention to treat and as treated populations.

\section{Discussion}

\section{Trial status}

The trial is now actively recruiting at centers in the United Kingdom, France and Germany.

Through investigator meetings and feedback, a few recurrent operational issues have arisen. Many centers have an admission for completion of all TAVI-related tests prior to a multidiscliplinary meeting decision at a later date. This then requires a further attendance to hospital for consent, baseline assessments and randomization. This can be expedited if a Heart Team can convene for a decision while the patient is an inpatient so that they can be approached, consented, etcetera. Regarding the consent process, this target population is often rather elderly and may, quite appropriately, be apprehensive regarding the TAVI procedure. So investigators must carefully and simply explain the trial and the consent process. Care must also be taken in assessment of the level of angina given its importance in the inclusion/exclusion criteria.

We are optimistic that the trial has achievable enrollment targets and will answer an important question in this nascent field.

\footnotetext{
Abbreviations

AKIN: Acute kidney injury network; AS: Aortic stenosis; BMS: Bare metal stent; CABG: Coronary artery bypass grafting; CAD: Coronary artery disease; Cx: Circumflex artery; DES: Drug eluting stent; DSE: Dobutamine stress echocardiography; LAD: Left anterior descending artery; LMS: Left main stem; LV: Left ventricular; LVEF: Left ventricular ejection fraction; MACCE: Major adverse cardiovascular or cerebrovascular event; MDT: Multidisciplinary team; MI: Myocardial infarction; PCl: Percutaneous coronary intervention; RCA: Right coronary artery; RCT: Randomized controlled trial; sAVR: Surgical aortic valve replacement; SVG: Saphenous vein graft; TAVI: Transcatheter aortic valve implantation; TIA: Transient ischemic attack; TTE: Transthoracic echocardiography; TOE: Transesophageal echocardiography; VARC: Valve academic research consortium; VARC2: Valve academic research consortium second guidelines.
}

\section{Competing interests}

Professor Redwood and Dr. Thomas are proctors for Edwards Lifesciences. Dr. Khawaja is in receipt of a British Heart Foundation Clinical Research Training Fellowship FS/12/15/29380.

\section{Authors' contributions}

MZK, SRR and MRT developed and designed the trial. MZK, DW and SP are responsible for the power calculation and statistical design and integrity of the protocol. All authors have read and approved the final manuscript.

\section{Acknowledgements}

The trial is sponsored by Guy's \& St Thomas' NHS Foundation Trust via educational grants from Edwards Lifesciences (Irvine, CA, USA) and Boston Scientific (Nantick, MA. USA). The conception, design, conduct and dissemination of the trial and its results is independent of the funding organizations.

\section{Author details}

${ }^{1}$ The Rayne Institute, Cardiovascular Division, King's College London, BHF Centre of Excellence, St Thomas' Hospital, Westminster Bridge Road, London SE1 7EH, UK. 'Department of Cardiology, Guy's \& St Thomas' NHS Foundation Trust, Westminster Bridge Road, London SE1 7EH, UK. ${ }^{3}$ Department of Clinical Sciences, Liverpool School of Tropical Medicine, Pembroke PI, Liverpool, Merseyside L3 5QA, UK. ${ }^{4}$ Department of Medical Statistics, London School of Hygiene and Tropical Medicine, Keppel St, Bloomsbury, London WC1E 7HT, UK.

Received: 17 January 2014 Accepted: 3 July 2014

Published: 24 July 2014

\section{References}

1. Cribier A, Eltchaninoff H, Bash A, Borenstein N, Tron C, Bauer F, Derumeaux G, Anselme F, Laborde F, Leon MB: Percutaneous transcatheter implantation of an aortic valve prosthesis for calcific aortic stenosis: first human case description. Circulation 2002, 106:3006-3008.

2. Ortlepp JR, Schmitz F, Bozoglu T, Hanrath P, Hoffmann R: Cardiovascular risk factors in patients with aortic stenosis predict prevalence of coronary artery disease but not of aortic stenosis: an angiographic pair matched case-control study. Heart 2003, 89:1019-1022.

3. Dworakowski R, MacCarthy PA, Monaghan M, Redwood S, El-Gamel A, Young C, Bapat V, Hancock J, Wilson K, Brickham B, Wendler O, Thomas MR: Transcatheter aortic valve implantation for severe aortic stenosis-a new paradigm for multidisciplinary intervention: a prospective cohort study. Am Heart J 2010, 160:237-243.

4. Masson J-B, Lee M, Boone RH, Ali Al A, Bugami Al S, Hamburger J, John Mancini GB, Ye J, Cheung A, Humphries KH, Wood D, Nietlispach F, Webb JG: Impact of coronary artery disease on outcomes after transcatheter aortic valve implantation. Catheter Cardiovasc Interv 2010, 76:165-173.

5. Dewey TM, Brown DL, Herbert MA, Culica D, Smith CR, Leon MB, Svensson LG, Tuzcu M, Webb JG, Cribier A, Mack MJ: Effect of concomitant coronary artery disease on procedural and late outcomes of transcatheter aortic valve implantation. Ann Thorac Surg 2010, 89:758-767. discussion 767.

6. Mohler ER, Sheridan MJ, Nichols R, Harvey WP, Waller BF: Development and progression of aortic valve stenosis: atherosclerosis risk factors-a causal relationship? a clinical morphologic study. Clin Cardiol 1991, 14:995-999.

7. Gerckens U, Latsios G, Mueller R, Buellesfeld L, Sauren B, Iversen S, Felderhof T, Grube E: Left main PCl after trans-subclavian CoreValve implantation: successful outcome of a combined procedure for management of a rare complication. Clin Res Cardiol 2009, 98:687-690.

8. Geist V, Sherif MA, Khattab AA: Successful percutaneous coronary intervention after implantation of a CoreValve percutaneous aortic valve. Catheter Cardiovasc Interv 2009, 73:61-67.

9. Sohal M, Stevens K, Rajani R, Khawaja MZ, De Belder A, Hildick-Smith D: Percutaneous coronary intervention of the right coronary artery following deployment of the CoreValve ${ }^{\circledast}$ revalving system. Eurolntervention 2009, 5:5. online [http://www.pcronline.com/eurointervention/23rd_issue/case4/]. 'doi:10.1074/jbc.R4000028200' or 'e8278'.

10. Leon MB, Kodali S, Williams M, Oz M, Smith C, Stewart A, Schwartz A, Collins M, Moses JW: Transcatheter aortic valve replacement in patients with critical aortic stenosis: rationale, device descriptions, early clinical experiences, and perspectives. Semin Thorac Cardiovasc Surg 2006, 18:165-174. 
11. Varadarajan P, Kapoor N, Bansal RC, Pai RG: Clinical profile and natural history of 453 nonsurgically managed patients with severe aortic stenosis. Ann Thorac Surg 2006, 82:2111-2115

12. Monin J-L, Quéré J-P, Monchi M, Petit H, Baleynaud S, Chauvel C, Pop C, Ohlmann P, Lelguen C, Dehant P, Tribouilloy C, Guéret P: Low-gradient aortic stenosis: operative risk stratification and predictors for long-term outcome: a multicenter study using dobutamine stress hemodynamics. Circulation 2003, 108:319-324.

13. Quere J-P, Monin J-L, Levy F, Petit H, Baleynaud S, Chauvel C, Pop C, Ohlmann P, Lelguen C, Dehant P, Gueret P, Tribouilloy C: Influence of preoperative left ventricular contractile reserve on postoperative ejection fraction in low-gradient aortic stenosis. Circulation 2006, 113:1738-1744.

14. Davies JE, Whinnett ZI, Francis DP, Manisty CH, Aguado-Sierra J, Willson K, Foale RA, Malik IS, Hughes AD, Parker KH, Mayet J: Evidence of a dominant backward-propagating "suction" wave responsible for diastolic coronary filling in humans, attenuated in left ventricular hypertrophy. Circulation 2006, 113:1768-1778.

15. Davies JE, Sen S, Broyd C, Hadjiloizou N, Baksi J, Francis DP, Foale RA, Parker KH, Hughes AD, Chukwuemeka A, Casula R, Malik IS, Mikhail GW, Mayet J: Arterial pulse wave dynamics after Percutaneous Aortic valve replacement: fall in coronary diastolic suction with increasing heart rate as a basis for angina symptoms in Aortic Stenosis. Circulation 2011, 124:1565-1572.

16. Levine GN, Bates ER, Blankenship JC, Bailey SR, Bittl JA, Cercek B, Chambers CE, Ellis SG, Guyton RA, Hollenberg SM, Khot UN, Lange RA, Mauri L, Mehran R, Moussa ID, Mukherjee D, Nallamothu BK, Ting HH: 2011 ACCF/AHA/SCAI Guideline for Percutaneous Coronary Intervention: a report of the American College of Cardiology Foundation/American Heart Association Task Force on Practice Guidelines and the Society for Cardiovascular Angiography and Interventions. Circulation 2011, 124:e574-e651.

17. Thygesen K, Alpert JS, Jaffe AS, Simoons ML, Chaitman BR, White HD, Joint ESC/ACCF/AHA/WHF Task Force for the Universal Definition of Myocardial: Third universal definition of myocardial infarction. Eur Heart J 2012, 33:2551-2567

18. Cutlip DE, Baim DS, Ho KK, Popma JJ, Lansky AJ, Cohen DJ, Carrozza JP, Chauhan MS, Rodriguez O, Kuntz RE: Stent thrombosis in the modern era: a pooled analysis of multicenter coronary stent clinical trials. Circulation 2001, 103:1967-1971.

19. Moreno R, Fernández C, Hernández R, Alfonso F, Angiolillo DJ, Sabaté M, Escaned J, Bañuelos C, Fernández-Ortiz A, Macaya C: Drug-eluting stent thrombosis: results from a pooled analysis including 10 randomized studies. J Am Coll Cardiol 2005, 45:954-959.

20. Doyle BJ, Rihal CS, Gastineau DA, Holmes DR: Bleeding, blood transfusion, and increased mortality after percutaneous coronary intervention: implications for contemporary practice. J Am Coll Cardio/ 2009, 53:2019-2027.

21. Rao SV, Eikelboom J, Steg PG, Lincoff AM, Weintraub WS, Bassand J-P, Rao AK, Gibson CM, Petersen JL, Mehran R, Manoukian SV, Charnigo R, Lee KL, Moscucci M, Harrington RA, Academic Bleeding Consensus Multidisciplinary Working Group: Standardized reporting of bleeding complications for clinical investigations in acute coronary syndromes: a proposal from the academic bleeding consensus $(A B C)$ multidisciplinary working group. Am Heart J 2009, 158:881-886.e1.

22. Ranucci M, Ballotta A, Kunkl A, De Benedetti D, Kandil H, Conti D, Mollichelli N Bossone E, Mehta RH: Influence of the timing of cardiac catheterization and the amount of contrast media on acute renal failure after cardiac surgery. Am J Cardiol 2008, 101:1112-1118.

23. Abdel-Wahab M, Zahn R, Horack M, Gerckens U, Schuler G, Sievert H, Naber C, Voehringer M, Schäfer U, Senges J, Richardt G: Transcatheter aortic valve implantation in patients with and without concomitant coronary artery disease: comparison of characteristics and early outcome in the German multicenter TAVI registry. Clin Res Cardiol 2012, 101:973-981.

24. Ussia GP, Barbanti M, Colombo A, Tarantini G, Petronio AS, Ettori F, Ramondo A, Santoro G, Klugmann S, Bedogni F, Antoniucci D, Maisano F, Marzocchi A, Poli A, De Carlo M, Fiorina C, De Marco F, Napodano M, Violini R, Bortone AS, Tamburino C, CoreValve Italian Registry Investigators: Impact of coronary artery disease in elderly patients undergoing transcatheter aortic valve implantation: Insight from the Italian CoreValve Registry. Int J Cardiol 2013, 167:943-950.

25. D'Ascenzo F, Conrotto F, Giordana F, Moretti C, D'Amico M, Salizzoni S, Omedè P, La Torre M, Thomas M, Khawaja Z, Hildick-Smith D, Ussia G, Barbanti M, Tamburino C, Webb J, Schnabel RB, Seiffert M, Wilde S, Treede H, Gasparetto V, Napodano M, Tarantini G, Presbitero P, Mennuni M, Rossi ML,
Gasparini M, Biondi Zoccai G, Lupo M, Rinaldi M, Gaita F, et al: Mid-term prognostic value of coronary artery disease in patients undergoing transcatheter aortic valve implantation: a meta-analysis of adjusted observational results. Int J Cardio/ 2013, 168:2528-2532.

26. Abdel-Wahab M, Mostafa AE, Geist V, Stöcker B, Gordian K, Merten C, Richardt D, Toelg R, Richardt G: Comparison of outcomes in patients having isolated transcatheter aortic valve implantation versus combined with preprocedural percutaneous coronary intervention. Am J Cardiol 2012, 109:581-586

27. Goel SS, Agarwal S, Tuzcu EM, Ellis SG, Svensson LG, Zaman T, Bajaj N, Joseph L, Patel NS, Aksoy O, Stewart WJ, Griffin BP, Kapadia SR: Percutaneous coronary intervention in patients with severe aortic stenosis: implications for transcatheter aortic valve replacement. Circulation 2012, 125:1005-1013.

28. Leon MB, Smith CR, Mack M, Miller DC, Moses JW, Svensson LG, Tuzcu EM, Webb JG, Fontana GP, Makkar RR, Brown DL, Block PC, Guyton RA, Pichard AD, Bavaria JE, Herrmann HC, Douglas PS, Petersen JL, Akin JJ, Anderson WN, Wang D, Pocock S, PARTNER Trial Investigators: Transcatheter aortic-valve implantation for aortic stenosis in patients who cannot undergo surgery. N Engl J Med 2010, 363:1597-1607.

29. Smith CR, Leon MB, Mack MJ, Miller DC, Moses JW, Svensson LG, Tuzcu EM, Webb JG, Fontana GP, Makkar RR, Williams M, Dewey T, Kapadia S, Babaliaros V, Thourani VH, Corso P, Pichard AD, Bavaria JE, Herrmann HC, Akin JJ, Anderson WN, Wang D, Pocock SJ: Transcatheter versus surgical aortic-valve replacement in high-risk patients. N Engl J Med 2011, 364:2187-2198

30. Thomas M, Schymik G, Walther T, Himbert D, Lefèvre T, Treede H, Eggebrecht $\mathrm{H}$, Rubino P, Colombo A, Lange R, Schwarz RR, Wendler O: One-Year Outcomes of Cohort 1 in the Edwards SAPIEN Aortic Bioprosthesis European outcome (SOURCE) registry. Circulation 2011, 124:425-433.

31. Moat NE, Ludman $P$, De Belder MA, Bridgewater $B$, Cunningham AD, Young CP, Thomas M, Kovac J, Spyt T, MacCarthy PA, Wendler O, Hildick-Smith D, Davies SW, Trivedi U, Blackman DJ, Levy RD, Brecker SJD, Baumbach A, Daniel T, Gray H, Mullen MJ: Long-term outcomes after transcatheter aortic valve implantation in high-risk patients with severe aortic stenosis: the U.K. TAVI (United Kingdom Transcatheter Aortic Valve Implantation) Registry. J Am Coll Cardiol 2011, 58:2130-2138.

32. Kodali SK, Williams MR, Smith CR, Svensson LG, Webb JG, Makkar RR, Fontana GP, Dewey TM, Thourani VH, Pichard AD, Fischbein M, Szeto WY, Lim S, Greason KL, Teirstein PS, Malaisrie SC, Douglas PS, Hahn RT, Whisenant B, Zajarias A, Wang D, Akin JJ, Anderson WN, Leon MB, PARTNER Trial Investigators: Two-year outcomes after transcatheter or surgical aortic-valve replacement. N Engl J Med 2012, 366:1686-1695.

33. Leon MB, Piazza N, Nikolsky E, Blackstone EH, Cutlip DE, Kappetein A-P, Krucoff MW, Mack M, Mehran R, Miller C, Morel M-A, Petersen J, Popma JJ, Takkenberg JJM, Vahanian A, Van Es G-A, Vranckx P, Webb JG, Windecker S, Serruys PW: Standardized endpoint definitions for Transcatheter Aortic Valve Implantation clinical trials: a consensus report from the Valve Academic Research Consortium. J Am Coll Cardiol 2011, 57:253-269.

34. Kappetein AP, Head SJ, Genereux P, Piazza N, Van Mieghem NM, Blackstone EH, Brott TG, Cohen DJ, Cutlip DE, Van Es G-A, Hahn RT, Kirtane AJ, Krucoff MW, Kodali S, Mack MJ, Mehran R, Rodés-Cabau J, Vranckx P, Webb JG, Windecker S, Serruys PW, Leon MB: Updated standardized endpoint definitions for transcatheter aortic valve implantation: the Valve Academic Research Consortium-2 consensus document. Eur Heart J 2012, 33:2403-2418.

35. Pocock SJ, Ariti CA, Collier TJ, Wang D: The win ratio: a new approach to the analysis of composite endpoints in clinical trials based on clinical priorities. Eur Heart J 2012, 33:176-182.

doi:10.1186/1745-6215-15-300

Cite this article as: Khawaja et al:: The percutaneous coronary intervention prior to transcatheter aortic valve implantation (ACTIVATION) trial: study protocol for a randomized controlled trial. Trials 2014 15:300. 